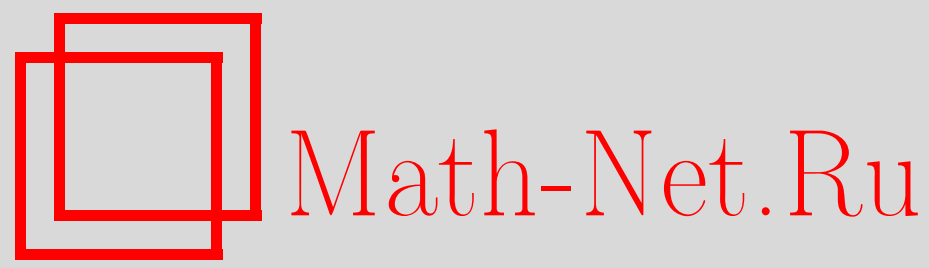

В. В. Рыжиков, Типичность изоморфизма сохраняющих меру преобразований при изоморфизме их декартовых степеней, Матем. заметки, 1996, том 59, выпуск 4, 630-632

DOI: https://doi.org/10.4213/mzm1757

Использование Общероссийского математического портала MathNet.Ru подразумевает, что вы прочитали и согласны с пользовательским соглашением

http://www. mathnet.ru/rus/agreement

Параметры загрузки:

IP : 3.91 .87 .62

26 апреля 2023 г., $10: 24: 28$

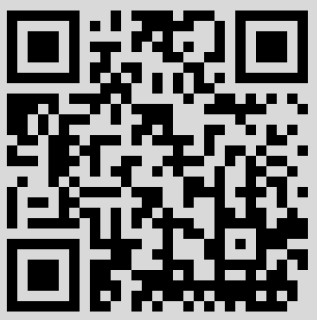




\section{ТИПИЧНОСТЬ ИЗОМОРФИЗМА СОХРАНЯЮЩИХ МЕРУ ПРЕОБРАЗОВАНИЙ ПРИ ИЗОМОРФИЗМЕ ИХ ДЕКАРТОВЫХ СТЕПЕНЕЙ}

\section{В. В. Рыжиков}

А. М. Стёпин (со ссылкой на Ж.-П. Тувено) обратил внимание автора на следующую задачу из эргодической теории. Пусть $S$ и $T$ - сохраняющие меру обратимые преобразования вероятностного пространства Лебега $(X, \mu)$ и известно, что их декартовы квадраты $S \times S$ и $T \times T$ метрически изоморфны. Последнее означает, что для некоторого $\Phi$, сохраняющего меру $\mu \times \mu$ обратимого преобразования $X \times X$, выполнено

$$
\Phi(S \times S) \Phi^{-1}=T \times T .
$$

Будут ли изоморфны $S$ и $T$ ?

Можно поставить также аналогичную задачу при условии изоморфизма декартовых степеней порядка $d>2$. В связи с этим возникает следующее понятие.

ОПРЕДЕЛЕНИЕ. Говорим, что преобразование $T$ является $d$-стабильным, $d>1$, если для любого $S$ изоморфизм $S \times \cdots \times S$ и $T \times \cdots \times T$ ( $d$ сомножителей) влечет изоморфизм $S$ и $T$.

\section{В заметке доказьвается следующая}

TEOPEмA. B әруппе $\operatorname{Aut}(\mu)$ всех сохраняющих меру преобразований $(X, \mu)$ свойство d-стабильности есть типичное свойство.

Напомним, что свойство преобразования назьвается типичным, если множество преобразований, удовлетворяющих этому свойству, есть множество второй категории относительно (метризуемой) топологии слабой сходимости в $\operatorname{Aut}(\mu)$. Следующие примеры типичных свойств установлены Рохлиным и Халмошем. Будем обозначать одним и тем же символом $T$ преобразование и унитарный оператор в $L_{2}(X, \mu)$, соответствующий этому преобразованию: $(T f)(x)=f(T x)$. (Такой оператор задает автоморфизм унитарного кольца.) Свойства

$$
\exists r(i) \rightarrow \infty \quad T^{r(i)} \rightarrow E
$$

и

$$
\exists m(i) \rightarrow \infty \quad T^{m(i)} \rightarrow \Theta
$$

где $E$ - тождественный оператор, а $\Theta$ - оператор ортопроекции на пространство констант $L_{2}(X, \mu)$, являются типичными.

В настоящей заметке существенно используется понятие $\varkappa$-перемешивания - об этом инварианте динамических систем см. [1], [2]. Результат, которым мы будем пользоваться, доказан в [2] и формулируется следующим образом:

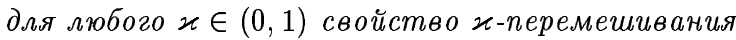

$$
\exists n(i) \rightarrow \infty \quad T^{n(i)} \rightarrow(1-\varkappa) E+\varkappa \Theta
$$

является типичным свойством.

ЗАмЕчАниЕ. В работе [2] были предъявлены примеры $\varkappa$-перемешивающих потоков для всех $\varkappa \in(1 / 2,1)$. Используя технику построения потоков с циклической аппроксимацией (потоков ранга 1 ), автор настоящей заметки построил примеры таких потоков для всех $\varkappa \in(0,1)$. 
Лемма. Пусть $T-\varkappa$-перемешиваюший автоморфизм, $0<\varkappa<1, u$ для автоморфизма $S$ выполнено

$$
\Phi(S \otimes \cdots \otimes S) \Phi^{-1}=T \otimes \cdots \otimes T,
$$

где $\Phi$ - оператор, отвечающий преобразованию пространства $X \times \cdots \times X$, сохраняюшего меру $\mu \otimes \cdots \otimes \mu$. Тогда $S$ и т изоморфны.

ДоКАЗАТЕЛЬство. Рассмотрим подробно случай $d=2$. При $d>2$ рассуждения аналогичны. Пусть для оператора $\Phi$, отвечающего автоморфизму $(X \times X, \mu \otimes \mu)$, выполнено равенство

$$
\Phi(S \otimes S) \Phi^{-1}=T \otimes T .
$$

Преобразование $T \times T$ обладает двумя координатными факторами, которые под действием $\Phi$ переходят в некоторые факторы преобразования $S \times S$. (Напомним, что фактором назьвается ограничение преобразования на инвариантную $\sigma$-подалгебру алгебры всех измеримых множеств.) Координатные алгебры образованы множествами вида $X \times A$ и множествами вида $A \times X$ : им соответствуют пространства $\mathbf{1} \otimes L_{2}(X, \mu)$ и $L_{2}(X, \mu) \otimes \mathbf{1}$.

Наша цель - доказать, что $\Phi$ переводит координатную алгебру в координатную. Тем самым, очевидно, будет осушествлен изоморфизм между $S$ и $T$. Для этого в нашем случае достаточно установить, что образ пространства $1 \otimes L_{2}(X, \mu)$ под действием $\Phi$ совпадает с пространством $1 \otimes L_{2}(X, \mu)$ или $L_{2}(X, \mu) \otimes \mathbf{1}$

Пусть при $\varkappa \in(0,1)$ выполнено $T^{n(i)} \rightarrow(1-\varkappa) E+\varkappa \Theta$. Докажем, что при выполнении условий леммы имеет место

$$
S^{n(i)} \rightarrow(1-\varkappa) E+\varkappa \Theta .
$$

Имеем

$$
\Phi(S \otimes S)^{n(i)} \Phi^{-1} \rightarrow((1-\varkappa) E+\varkappa \Theta) \otimes((1-\varkappa) E+\varkappa \Theta) .
$$

$\mathrm{C}$ другой стороны, для некоторой подпоследовательности $k\left(i^{\prime}\right)$ получим

$$
(S \otimes S)^{k\left(i^{\prime}\right)} \rightarrow Q \otimes Q .
$$

Учитывая, что $E \otimes E$ и $\Theta \otimes \Theta$ коммутируют с $\Phi$, находим

$$
Q \otimes Q=(1-\varkappa)^{2}(E \otimes E)+\varkappa^{2}(\Theta \otimes \Theta)+\varkappa(1-\varkappa) \Phi^{-1}((E \otimes \Theta)+(\Theta \otimes E)) \Phi .
$$

Таким образом, для любой неотрицательной функции $f \in L_{2}(X, \mu)$ вьполнены неравенства

$$
Q f \otimes Q f \geqslant(1-\varkappa)^{2} f \otimes f, \quad Q f \otimes Q f \geqslant \varkappa^{2} \Theta f \otimes \Theta f .
$$

Из неравенств вытекает, что

$$
Q f \geqslant(1-\varkappa) f, \quad Q f \geqslant \varkappa \Theta f,
$$

следовательно, в силу произвольности положительной функции $f$ получаем

$$
Q=(1-\varkappa) E+\varkappa \Theta .
$$

Пусть $H \subset L_{2}(X, \mu)$ обозначает пространство функций с нулевым средним. Тогда имеет место разложение

$$
L_{2}(X \times X, \mu \otimes \mu)=\{\text { Const }\} \oplus(H \otimes \mathbf{1}) \oplus(\mathbf{1} \otimes H) \oplus(H \otimes H),
$$

где $\{$ Const $\}$ обозначает пространство постоянных функций. Заметим, что

$$
\Phi(H \otimes \mathbf{1}) \subseteq(H \otimes \mathbf{1}) \oplus(\mathbf{1} \otimes H) .
$$


Действительно, последовательность $(T \otimes T)^{n(i)}$ сходится на $(H \otimes \mathbf{1})$ к оператору $(1-\varkappa)(E \otimes E)$, следовательно, последовательность $(T \otimes T)^{n(i)}=\Phi(S \otimes S)^{n(i)} \Phi^{-1}$ сходится на $\Phi(H \otimes 1)$ также к оператору $(1-\varkappa)(E \otimes E)$. Это возможно лише в случае, когда выполнено (1), так как на $(\boldsymbol{H} \otimes \boldsymbol{H})$ эти последовательности операторов сходятся к $(1-\varkappa)^{2}(E \otimes E)$, но $(1-\varkappa)^{2}<(1-\varkappa)$. Нам осталось лишь показать, что в действительности имеет место

$$
\Phi(H \otimes \mathbf{1}) \subseteq(H \otimes \mathbf{1}) \quad \text { или } \quad \Phi(H \otimes \mathbf{1}) \subseteq(\mathbf{1} \otimes H) .
$$

Для любой ограниченной функции $f \in H$ существуют функции $f_{1}$ и $f_{2}$ такие, что

$$
\Phi(f \otimes \mathbf{1})=a\left(f_{1} \otimes \mathbf{1}\right)+b\left(\mathbf{1} \otimes f_{2}\right) .
$$

Из того, что пространство $\left.\Phi\left(L_{2}(X, \mu)\right) \otimes \mathbf{1}\right)$ соответствует фактору и замкнуто относительно произведения ограниченных функций, вытекает

$$
(\Phi(f \otimes \mathbf{1}))^{2} \in(H \otimes \mathbf{1}) \oplus(\mathbf{1} \otimes H) \oplus\{\text { Const }\} .
$$

Из (3) и (4) следует

$$
a b\left(f_{1} \otimes f_{2}\right) \in(H \otimes \mathbf{1}) \oplus(\mathbf{1} \otimes H) \oplus\{\text { Const }\},
$$

поэтому $a b=0$, или $f_{1}$ есть константа, или $f_{2}$ есть константа. Таким образом, мы показали, что

$$
\Phi(H \otimes \mathbf{1}) \subset(H \otimes \mathbf{1}) \cup(\mathbf{1} \otimes H) .
$$

Так как $H \otimes \mathbf{1}$ и $1 \otimes H$ имеют нулевое пересечение а $\Phi(H \otimes \mathbf{1})$ есть линейное пространство, получим (2). Пусть, например, имеет место $\Phi(H \otimes \mathbf{1}) \subseteq(H \otimes \mathbf{1})$. Повторим рассуждения, поменяв местами $S$ и $T$. Тогда получим $\Phi^{-1}(H \otimes \mathbf{1}) \subseteq(H \otimes \mathbf{1})$. Эти включения вместе дают равенство $\Phi(H \otimes \mathbf{1})=(H \otimes \mathbf{1})$ и требуемый изоморфизм координатных факторов.

В общем случае аналогичные рассуждения приводят к

$$
\Phi(f \otimes \mathbf{1} \otimes \cdots \otimes \mathbf{1}) \in(H \otimes \mathbf{1} \otimes \cdots \otimes \mathbf{1}) \oplus \cdots \oplus(\mathbf{1} \otimes \cdots \otimes \mathbf{1} \otimes H)
$$

и

$$
(\Phi(f \otimes \mathbf{1} \otimes \cdots \otimes \mathbf{1}))^{2} \in(H \otimes \mathbf{1} \otimes \cdots \otimes \mathbf{1}) \oplus \cdots \oplus(\mathbf{1} \otimes \cdots \otimes \mathbf{1} \otimes H) \oplus\{\text { Const }\},
$$

и, окончательно, к изоморфизму координатных факторов.

Теорема вытекает из леммы и типичности свойства $\varkappa$-перемешивания.

В эргодической теории является открытым вопрос о типичности включения автоморфизма в поток. В связи с этим сформулируем следующую задачу. Оператор $P$ на $L_{2}(X, \mu)$ назьвается бистохастическим, если $P \mathbf{1}=P^{*} \mathbf{1}=1$ и операторы $P, P^{*}$ являются положительными в том смысле, что они неотрицательные функции переводят в неотрицательные. (Операторы $T, E$ и $\Theta$ являются бистохастическими.) На полугруппе положительных операторов задается порядок: $P>J$, если для любой положительной функции $f$ выполнено $P f>J f$. Определим следующее свойство автоморфизма $T$ : для любого бистохастического оператора $P$, коммутирующего с оператором $T$, для некоторого $a>0$ выполнено

$$
P^{*} P>a E \text { или } P^{*} P>a \Theta .
$$

Является ли это свойство типичным? Автор предполагает, что ответ утвердительный. Из утвердительного ответа вытекает нетипичность включения автоморфизма в поток.

Московский государственный университет им. М.В. Ломоносова

E-mail: vryz@math.math.msu.su 20.01 .95

\section{СПИСОК ЦИТИРОВАННОЙ ЛИТЕРАТУРЫ}

1. Оселедец В. И. // Матем. заметки 1969. Т. 5. №3. С. 323-326. 2. Стёпин А. М. // Изв. АН СССР. Сер. матем. 1986. Т. 50. №4. С. 801-834. 